\title{
Géométrie plane: Une axiomatique centrée sur la distance
}

\author{
Christoph Soland \\ Christoph Soland a obtenu en 1972 une licence en mathématiques à l'Université de \\ Lausanne et enseigne les mathématiques au Gymnase depuis 1979 . En 1997 il a écrit \\ une thèse sur les ovales de Descartes sous la direction de Oscar Burlet. Il s'intéresse \\ aux fondements des mathématiques, à l'axiomatique et, surtout, à la géométrie.
}

\section{Introduction}

Des points, leurs distances, et rien d'autre! C'est l'objectif que je me suis fixé pour concevoir une nouvelle axiomatique de la géométrie euclidienne plane. Dans cet esprit, Blumenthal [2] va beaucoup plus loin: il décrit en détail ce qui différencie les espaces euclidiens des autres espaces métriques, notamment à l'aide des déterminants de Cayley-Menger. Mais le cas du plan euclidien nécessite moins de moyens; je propose ici un traitement léger et rapide.

Je développe le minimum de concepts et de théorèmes permettant de montrer que la nouvelle axiomatique est équivalente à celle de Hilbert, en mettant l'accent sur l'aire, la mesure des angles et leur additivité.

J'utilise surtout des fonctions polynomiales ou rationnelles dont les variables sont des distances: la formule de Héron et sa généralisation, le déterminant de Cayley-Menger, le théorème du cosinus et la relation de Stewart.

Bekanntlich hat Euklid (um 300 v.Chr.) als erster versucht, die Geometrie auf eine axiomatische Grundlage zu stellen; aber erst mit dem Erscheinen von Hilberts „Grundlagen der Geometrie“ (1899) lag ein hieb- und stichfestes Axiomensystem vor. Im Gegensatz zu den Axiomen für algebraische Strukturen sind die Axiome der Geometrie von grosser Zahl und unübersichtlich. Nach Hilbert sind daher verschiedene andere Ansätze zur Axiomatisierung versucht worden, so z.B. der Aufbau der Geometrie aus dem Spiegelungsbegriff oder aus dem Kollineationsbegriff. Unser Autor nimmt im Anschluss an Blumenthal den Distanzbegriff als Grundlage, und er kommt mit sechs Axiomen aus, wovon die ersten drei die üblichen Axiome eines metrischen Raumes sind. Die „quadratische Natur" der Distanz macht es allerdings nötig, die beim weiteren Fortschreiten auftretenden Polynome mit Hilfe von Computeralgebra in Faktoren zu zerlegen. 
Le calcul est au service du sens. J'ai donc choisi de laisser la manipulation des expressions algébriques à un logiciel de calcul formel (abrégé LCF dans la suite), dans la mesure où cette manipulation n'éclaire pas le propos.

Les notations sont classiques pour la plupart: $|a b|$ désigne la distance des points a et $\mathrm{b} ;(\mathrm{abc} \ldots \mathrm{z})$ une liste ordonnée de points et $\{\mathrm{abc} \ldots \mathrm{z}\}$ l'ensemble non ordonné de ces mêmes points. Enfin $(6: 2: 8: 4)=(9: 3: 12: 6)$ dénote deux listes proportionnelles de nombres.

Je définis dès à présent quelques fonctions des distances mutuelles de trois ou quatre points. Leur utilité apparaîtra en son temps.

Définition 1 (Quelques fonctions)

1. $C\{\mathrm{abc}\}, C\{\mathrm{abcd}\}$. Ce sont les déterminants de la proposition $1 \mathrm{infra}$;

2. $D(\mathrm{ab}, \mathrm{c}):=(|\mathrm{ac}|+|\mathrm{bc}|-|\mathrm{ab}|)(|\mathrm{ab}|+|\mathrm{bc}|-|\mathrm{ac}|)$;

3. $\kappa(a b c):=\left(|a b|^{2}+|b c|^{2}-|a c|^{2}\right) /(2|a b||b c|)$;

4. $S(\mathrm{abc}, \mathrm{x}):=|\mathrm{bc}||\mathrm{ax}|^{2}-|\mathrm{ca}||\mathrm{bx}|^{2}+|\mathrm{ab}||\mathrm{cx}|^{2}-|\mathrm{bc}||\mathrm{ca}||\mathrm{ab}|$.

\section{Genèse des axiomes}

Le plan euclidien est d'abord un espace métrique, les trois premiers axiomes sont donc ceux que vérifie n'importe quelle métrique. Le quatrième axiome est un important raccourci par rapport à [2] qui rend la droite isométrique à $\mathbb{R}$.

Mes deux derniers axiomes sont:

- quatre points coplanaires déterminent un tétraèdre de volume nul;

- il existe dans le plan un triangle d'aire non nulle (axiome de Hilbert HI3).

Le déterminant de Cayley-Menger [1], qui décrit le $n$-volume du simplexe de $\mathbb{R}^{n}$ en fonction des distances mutuelles de ses sommets, traduit ces axiomes en termes de distance. Je précise cette description pour les dimensions 2 et 3 :

\section{Proposition 1}

1. L'aire $\sigma\{\mathrm{abc}\}$ du triangle de sommets $\mathrm{a}, \mathrm{b}$, c est donnée par

$$
\sigma^{2}\{\mathrm{abc}\}=-\frac{1}{16} C\{\mathrm{abc}\},
$$

où

$$
\begin{aligned}
C\{\mathrm{abc}\}= & \left|\begin{array}{cccc}
0 & 1 & 1 & 1 \\
1 & 0 & |\mathrm{ab}|^{2} & |\mathrm{ac}|^{2} \\
1 & |\mathrm{ba}|^{2} & 0 & |\mathrm{bc}|^{2} \\
1 & |\mathrm{ca}|^{2} & |\mathrm{cb}|^{2} & 0
\end{array}\right| \\
= & (|\mathrm{ab}|+|\mathrm{ac}|+|\mathrm{bc}|)(|\mathrm{ab}|-|\mathrm{ac}|-|\mathrm{bc}|) \\
& \cdot(-|\mathrm{ab}|+|\mathrm{ac}|-|\mathrm{bc}|)(-|\mathrm{ab}|-|\mathrm{ac}|+|\mathrm{bc}|) .
\end{aligned}
$$


2. Le volume $\sigma\{\mathrm{abcd}\}$ du tétraèdre de sommets $\mathrm{a}, \mathrm{b}, \mathrm{c}, \mathrm{d}$ est donné par

$$
\sigma^{2}\{\text { abcd }\}=-\frac{1}{288} C\{\text { abcd }\}
$$

où

$$
C\{\mathrm{abcd}\}=\left|\begin{array}{ccccc}
0 & 1 & 1 & 1 & 1 \\
1 & 0 & |\mathrm{ab}|^{2} & |\mathrm{ac}|^{2} & |\mathrm{ad}|^{2} \\
1 & |\mathrm{ba}|^{2} & 0 & |\mathrm{bc}|^{2} & |\mathrm{bd}|^{2} \\
1 & |\mathrm{ca}|^{2} & |\mathrm{cb}|^{2} & 0 & |\mathrm{~cd}|^{2} \\
1 & |\mathrm{da}|^{2} & |\mathrm{db}|^{2} & |\mathrm{dc}|^{2} & 0
\end{array}\right|
$$

La preuve se trouve dans [1].

Si le premier déterminant de la proposition 1 se factorise aimablement et conduit (mutatis mutandis) à la formule de Héron bien connue $\sigma\{\mathrm{abc}\}=\sqrt{p(p-a)(p-b)(p-c)}$, le second, polynôme irréductible, se présente désagréablement comme une somme de vingtdeux monômes de degré six. Le LCF rend cependant sa manipulation facile.

\section{Enoncé des axiomes}

Soit $\mathrm{P}$ un ensemble et

$$
\text { distance }:(\mathrm{ab}) \mapsto|\mathrm{ab}|
$$

une application de $\mathrm{P}^{2}$ dans $\mathbb{R}$. Les distances deux à deux des points arbitraires $\mathrm{a}, \mathrm{b}, \mathrm{c}, \mathrm{d}$ vérifient les relations suivantes:

1. $|\mathrm{ab}|=0$ si, et seulement si $\mathrm{a}=\mathrm{b}$.

2. $|\mathrm{ab}|=|\mathrm{ba}|$.

3. $|\mathrm{ac}| \leq|\mathrm{ab}|+|\mathrm{bc}|$. (L'inégalité triangulaire.)

4. L'application $\mathrm{x} \mapsto|\mathrm{ax}|$ restreinte à n'importe quelle demi-droite d'extrémité a est une surjection de cette demi-droite sur $\mathbb{R}_{\geq 0}$. (Je n'utilise pas cet axiome avant la définition de la demi-droite (déf. 3).)

5. $C\{$ abcd $\}=0$.

6. Il existe trois points $\mathrm{p}, \mathrm{q}, \mathrm{r}$ tels que $C\{\mathrm{pqr}\} \neq 0$.

\section{Alignements}

Le deuxième concept après la distance est celui d'alignement qui caractérise les triples de points réalisant l'égalité dans l'inégalité triangulaire.

Définition 2 Un triple ordonné ( $\mathrm{abc}$ ) de points distincts est un alignement si $|\mathrm{ab}|+|\mathrm{bc}|=$ $|\mathrm{ac}|$. On dit que b est situé entre a et c. Trois points a, b, c sont colinéaires si l'une des permutations de (abc) est un alignement.

Dans un alignement ( $a b c)$, l'ordre des points est important. Si (abc) est un alignement, (cba) en est un aussi, mais les autres permutations de (abc) n'en sont pas. Les trois points sont en effet distincts et le système $|a b|+|b c|-|a c|=|a c|+|b c|-|a b|=0$ (par exemple) donne $|\mathrm{bc}|=0$ et $\mathrm{b}=\mathrm{c}($ axiome 1$)$. 
Trois points a, b, c sont colinéaires si leurs distances mutuelles annulent l'un des facteurs de $C\{a b c\}$. J'utilise ces facteurs pour définir la droite et ses intervalles.

Définition 3 Etant donné deux points a et $b$,

1. la droite $\mathrm{D}_{\mathrm{ab}}$ est l'ensemble des points $\mathrm{x}$ tels que $C(\mathrm{abx})=0$,

2. la demi-droite (fermée) $\mathrm{D}_{[\mathrm{ab} \infty}$ d'extrémité a passant $\mathrm{b}$ est l'ensemble des points $\mathrm{x}$ tels que $D(\mathrm{ab}, \mathrm{x})=0$,

3. le segment $\mathrm{S}_{[\mathrm{ab}]}$ est constitué de $\mathrm{a}, \mathrm{b}$ et de l'ensemble des points $\mathrm{x}$ situés entre $\mathrm{a}$ et $\mathrm{b}$.

L'axiome 4 se place logiquement ici. La restriction de l'application distance à une demidroite est en fait bijective. La preuve de cette proposition illustre l'emploi du LCF.

Proposition 2 L'application $\mathrm{x} \mapsto|\mathrm{ax}|$ restreinte à une demi-droite d'extrémité a est injective.

Preuve. Soit $\mathrm{x}$ et y deux points de la demi-droite $\mathrm{D}_{[\mathrm{ab} \infty}$ tels que $|\mathrm{ax}|=|\mathrm{ay}|$. On élimine $|a y|,|b x|$ et $|b y|$ dans le système

$$
\begin{aligned}
& C\{\mathrm{abxy}\}=0 \quad(\text { axiome 5), } \\
& D(\mathrm{ab}, \mathrm{x})=0, \quad D(\mathrm{ab}, \mathrm{y})=0 \quad\left(\mathrm{x}, \mathrm{y} \in \mathrm{D}_{[\mathrm{ab} \infty}\right), \\
& |\mathrm{ax}|-|\mathrm{ay}|=0 \quad \text { (hypothèse) }
\end{aligned}
$$

ce qui donne $|\mathrm{ab}|^{2}|\mathrm{xy}|^{4}=0$. Donc $|\mathrm{xy}|=0$ et l'on termine avec l'axiome 1 .

On a donc une bijection de $\mathrm{D}_{\text {[ab } \infty}$ sur les réels non négatifs, qui s'étend naturellement à une bijection de $\mathrm{D}_{\mathrm{ab}}$ sur l'ensemble des réels.

Définition 4 Soit $\mathrm{D}_{\mathrm{ab}}$ une droite et $\mathrm{x}$ un point arbitraire de $\mathrm{D}_{\mathrm{ab}}$. J'appelle coordonnée linéaire de $\mathrm{x}$ relativement au repère $(\mathrm{ab})$, notée $x_{(\mathrm{ab})}$ ou simplement $x$, le nombre réel $|\mathrm{ax}| /|\mathrm{ab}|$ si $\mathrm{x} \in \mathrm{D}_{[\mathrm{ab} \infty}$, le nombre $-|\mathrm{ax}| /|\mathrm{ab}|$ sinon.

Une demi-droite est déterminée par son extrémité et l'un de ses points, n'importe lequel. De même, une droite est déterminée par deux quelconques de ses points.

\section{Proposition 3}

1. Si $\mathrm{c}, \mathrm{c} \neq \mathrm{a}$, appartient à la demi-droite $\mathrm{D}_{[\mathrm{ab} \infty}$, alors $\mathrm{D}_{[\mathrm{ab} \infty}=\mathrm{D}_{[\mathrm{ac} \infty}$.

2. Si $\mathrm{c}, \mathrm{c} \neq \mathrm{a}$, appartient à la droite $\mathrm{D}_{\mathrm{ab}}$, alors $\mathrm{D}_{\mathrm{ab}}=\mathrm{D}_{\mathrm{ac}}$.

Preuve. Demi-preuve de 1. En éliminant $|\mathrm{bc}|$ et $|\mathrm{bx}|$ dans le système

$$
C\{\mathrm{abcx}\}=D(\mathrm{ab}, \mathrm{c})=D(\mathrm{ab}, \mathrm{x})=0,
$$

on obtient $|\mathrm{ab}|^{2} D^{2}(\mathrm{ac}, \mathrm{x})=0$. Donc $\mathrm{D}_{[\mathrm{ab} \infty} \subset \mathrm{D}_{[\mathrm{ac} \infty}$.

Demi-preuve de 2. En éliminant $|\mathrm{bc}|$ et $|\mathrm{bx}|$ dans le système

$$
C\{\mathrm{abcx}\}=C\{\mathrm{abc}\}=C\{\mathrm{abx}\}=0,
$$

on obtient $|\mathrm{ab}|^{4} C^{2}\{\mathrm{acx}\}=0$. Donc $\mathrm{D}_{\mathrm{ab}} \subset \mathrm{D}_{\mathrm{ac}}$.

Les inclusions réciproques se démontrent de la même façon. 


\section{Angles, triangles, mesures}

Le troisième concept important est celui d'angle et celui de sa mesure. Viennent ensuite le triangle et son aire.

Définition 5 J'appelle angle de sommet b tout triple ordonné (abc) de points distincts et triangle de sommets $\mathrm{a}, \mathrm{b}, \mathrm{c}$ tout triplet non ordonné $\{\mathrm{abc}\}$ de points distincts.

Je suppose connues les fonctions trigonométriques (définies par leurs séries), leurs inverses, ainsi que leurs propriétés élémentaires. [5] est une bonne réference. Je définis la mesure des angles via le théorème du cosinus [1].

Je m'assure d'abord que ces mesures sont des réels positifs, comme il se doit:

Lemme 4

$$
1-\kappa^{2}(\mathrm{abc})=\frac{-C\{\mathrm{abc}\}}{4|\mathrm{ab}|^{2}|\mathrm{bc}|^{2}} \geq 0 .
$$

Preuve. L'égalité résulte d'un calcul avec le LCF, l'inégalité de l'axiome 3 et de la factorisation de $C\{\mathrm{abc}\}$.

Définition 6 La mesure de l'angle (abc), notée $\mu(\mathrm{abc})$, est le nombre réel $\arccos (\kappa(\mathrm{abc}))$.

Puisque $-1 \leq \kappa(\mathrm{abc}) \leq 1$ (lemme 4$)$, les mesures d'angle appartiennent à l'intervalle $[0, \pi]$. Pour toute fonction $f$ j'abrégerai au besoin $f(\mu(\mathrm{abc}))$ par $f(\mathrm{abc})$.

Du point de vue axiomatique, c'est l'inégalité triangulaire qui rend non positif le déterminant $C\{a b c\}$, ouvrant la voie à la définition de l'aire.

Définition 7 L'aire du triangle $\{\mathrm{abc}\}$, notée $\sigma\{\mathrm{abc}\}$, est le nombre réel $\frac{1}{4}(-C\{\mathrm{abc}\})^{1 / 2}$.

On pourrait à ce stade traiter toute la trigonométrie du triangle et les cas d'isométrie de deux triangles. N'en faisons pas trop. . .

\section{Proposition 5}

$$
\cos (\mathrm{abc})=\kappa(\mathrm{abc})=\frac{|\mathrm{ab}|^{2}+|\mathrm{bc}|^{2}-|\mathrm{ac}|^{2}}{2|\mathrm{ab}||\mathrm{bc}|}, \quad \sin (\mathrm{abc})=\frac{(-C\{\mathrm{abc}\})^{1 / 2}}{2|\mathrm{ab}||\mathrm{bc}|} .
$$

Preuve. Ces formules résultent de la définition 6 et du lemme 4.

Théorème 6 Soit $\{\mathrm{abc}\}$ un triangle. Alors

1. $|\mathrm{ac}|^{2}=|\mathrm{ab}|^{2}+|\mathrm{bc}|^{2}-2|\mathrm{ab}||\mathrm{bc}| \cos (\mathrm{abc}) \quad$ (théorème du cosinus);

2. $\mu(\mathrm{cab})+\mu(\mathrm{abc})+\mu(\mathrm{bca})=\pi \quad$ (somme des angles $d u$ triangle).

Preuve. Le point 1. est une conséquence immédiate de la proposition 5.

Preuve de 2. La proposition 5 et une factorisation par le LCF donnent

$$
\cos (c a b) \cos (a b c)+\cos (b c a)=\sin (c a b) \sin (a b c) .
$$

Le théorème d'addition du cosinus donne alors

$$
\cos (\mu(\mathrm{cab})+\mu(\mathrm{abc}))=-\cos (\mathrm{bca})
$$

ou encore $\mu(\mathrm{cab})+\mu(\mathrm{abc})=\pi-\mu(\mathrm{bca})$. 


\section{Additivité des mesures}

Les propriétés fondamentales de l'aire et des mesures d'angle se résument en une configuration que j'appelle configuration de Stewart (Fig. 1), constituée d'un alignement et d'un quatrième point. J'ai besoin d'un lemme:

Lemme 7 Soit $(\mathrm{abc})$ un alignement et $\mathrm{x}$ un quatrième point. Alors $S(\mathrm{abc}, x)=0$.

Preuve. On élimine $|\mathrm{ac}|$ dans le système $C\{\mathrm{abcx}\}=0,|\mathrm{ab}|+|\mathrm{bc}|-|\mathrm{ac}|=0$, ce qui donne $S^{2}(\mathrm{abc}, \mathrm{x})=0$.

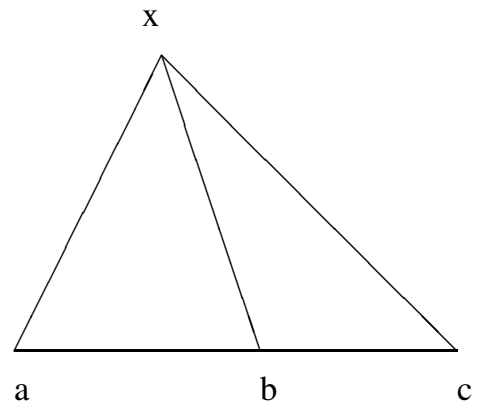

Fig. 1

Théorème 8 Soit $(\mathrm{abc})$ un alignement et $\mathrm{x}$ un quatrième point. Alors

1. $\mu(\operatorname{bax})=\mu(\operatorname{cax})$.

2. $\mu(\mathrm{abx})+\mu(\mathrm{cbx})=\pi=\mu(\mathrm{abc})$.

3. $\mu(\mathrm{axb})+\mu(\mathrm{bxc})=\mu(\mathrm{axc})$.

4. $\sigma\{\mathrm{axb}\}+\sigma\{\mathrm{bxc}\}=\sigma\{\mathrm{axc}\}$.

Preuve de 1. et 2 . Les numérateurs de $\cos (\mathrm{bax})-\cos (\mathrm{cax})$ et de $\cos (\mathrm{abx})+\cos (\mathrm{cbx})$ sont $\pm S(\mathrm{abc}, \mathrm{x})$. Ils sont donc nuls, puisque (abc) est un alignement.

Preuve de 3 . La somme des angles des triangles abx et cbx vaut $2 \pi$. Comme $\mu(\mathrm{abx})+$ $\mu(\operatorname{cbx})=\pi$, on en déduit que $\mu(\mathrm{axb})+\mu(\mathrm{cxb})=\pi-\mu(\mathrm{bax})-\mu(\mathrm{bcx})$. La somme des angles dans le triangle acx donne $\mu(\operatorname{axc})=\pi-\mu(\operatorname{cax})-\mu(\operatorname{acx})$. On termine avec le point 1 . de ce théorème: $\mu(\mathrm{bax})=\mu(\mathrm{cax})$ et $\mu(\mathrm{bcx})=\mu(\mathrm{acx})$.

Preuve de 4. Avec la relation de Stewart $S(\mathrm{abc}, \mathrm{x})=0$, j'élimine séparément (1) $|\mathrm{ax}|^{2},(2)$ $|c x|^{2}$ et (3) $|\mathrm{bx}|^{2}$ dans le numérateur de $\cos (\mathrm{axb}) \cos (\mathrm{bxc})-\cos (\mathrm{axc})$.

J'obtiens

1) $\cos (\mathrm{axb}) \cos (\mathrm{bxc})-\cos (\mathrm{axc})=\frac{4}{|\mathrm{ax}||\mathrm{bx}|^{2}|\mathrm{cx}|} * \frac{|\mathrm{ab}|}{|\mathrm{bc}|} * \sigma^{2}\{\mathrm{bcx}\}$

2) $\cos (a x b) \cos (b x c)-\cos (a x c)=\frac{4}{|a x||b x|^{2}|c x|} * \frac{|b c|}{|a b|} * \sigma^{2}\{a b x\}$, 
3) $\cos (\mathrm{axb}) \cos (\mathrm{bxc})-\cos (\mathrm{axc})=\frac{4}{|\mathrm{ax}||\mathrm{bx}|^{2}|\mathrm{cx}|} * \frac{|\mathrm{ab}||\mathrm{bc}|}{|\mathrm{ac}|^{2}} * \sigma^{2}\{\mathrm{acx}\}$.

En comparant les membres de droite de ces expressions, qui sont égaux, on voit que

$$
\begin{aligned}
(\sigma\{\mathrm{abx}\}: \sigma\{\mathrm{bcx}\}: \sigma\{\mathrm{acx}\}) & =(|\mathrm{ab}|:|\mathrm{bc}|:|\mathrm{ac}|)=(|\mathrm{ab}|:|\mathrm{bc}|:|\mathrm{ab}|+|\mathrm{bc}|) \\
& =(\sigma\{\mathrm{abx}\}: \sigma\{\mathrm{bcx}\}: \sigma\{\mathrm{abx}\}+\sigma\{\mathrm{bcx}\}) .
\end{aligned}
$$

Comme $\mu(\mathrm{abc})$ est indépendant du choix de $\mathrm{a} \in \mathrm{D}_{[\mathrm{ba} \infty}$ et de $\mathrm{c} \in \mathrm{D}_{[\mathrm{bc} \infty}$, on peut définir l'angle des demi-droites $\mathrm{D}_{[\mathrm{ba} \infty}, \mathrm{D}_{[\mathrm{bc} \infty}$ et sa mesure $\mu(\mathrm{abc})$.

\section{Retour à l'axiomatique}

Pour valider la nouvelle axiomatique, j'ai vérifié que ses axiomes impliquent les quinze axiomes plans de [4] traduits en annexe. Je laisse de côté la réciproque.

Tous les axiomes de Hilbert concernant les alignements, c'est-à-dire les axiomes HI1, HI2, HI3, HII1, HII2 et HII3 se prouvent à l'aide de la coordonnée (déf. 4).

Je démontre l'axiome de Pasch HII4 comme corollaire du théorème de Ménélaüs, luimême corollaire d'un théorème sur les aires. Il faut pour cela généraliser le théorème du cosinus (sans preuve) et définir le rapport de section.

Lemme 9 Soit deux droites B, C ayant un point commun a, repérées respectivement par (ab) et (ac), et soit $\varphi:=\mu(\mathrm{bac})$. Si $\mathrm{p}$ est un point de $\mathrm{B}$ de coordonnée $p=p_{(\mathrm{ab})}$ et $\mathrm{q}$ un point de $\mathrm{C}$ de coordonnée $q=q_{(\mathrm{ac})}$, alors

$$
|\mathrm{pq}|^{2}=p^{2}|\mathrm{ab}|^{2}+q^{2}|\mathrm{ac}|^{2}-2 p q|\mathrm{ab}||\mathrm{ac}| \cos \varphi .
$$

Définition 8 Soit $D_{a b}$ une droite de repère $(a b)$ et $p, q, r$ trois points distincts de $D_{a b}$. Alors le rapport de section (ou rapport) de $\mathrm{r}$ relativement au couple (pq), noté (pq,r), est le nombre réel

$$
(\mathrm{pq}, \mathrm{r}):=\frac{r-p}{r-q} .
$$

Relevons que le rapport (pq,r) ne dépend pas du choix du repère de $\mathrm{D}_{\mathrm{ab}}$ et qu'il est négatif exactement si (prq) est un alignement.

Théorème 10 (de Ménélaüs généralisé) Soit $\{\mathrm{abc}\}$ un triangle, p, q, r trois points situés respectivement sur les droites $\mathrm{D}_{\mathrm{bc}}, \mathrm{D}_{\mathrm{ca}}, \mathrm{D}_{\mathrm{ab}}$, et soit $\lambda:=(\mathrm{bc}, \mathrm{p}), \mu:=(\mathrm{ca}, \mathrm{q}), v:=$ $(\mathrm{ab}, \mathrm{r})$ les rapports de section respectifs que les points $\mathrm{p}, \mathrm{q}, \mathrm{r}$ déterminent avec les côtés $d u$ triangle $\{\mathrm{abc}\}$. Alors l'aire du triangle $\{\mathrm{pqr}\}$ vaut

$$
\sigma\{\mathrm{pqr}\}=\sigma\{\mathrm{abc}\} \frac{|\lambda \mu \nu-1|}{|\lambda-1||\mu-1||\nu-1|} .
$$

Preuve. ([3]) On exprime successivement en fonction des côtés du triangle $\{a b c\}$ :

- les cosinus des angles du triangle $\{a b c\}$,

- la coordonnée linéaire de $\mathrm{p}$ relativement au repère $(\mathrm{bc})$ et relativement au repère (cb):

$$
p_{1}=\lambda /(\lambda-1), \quad p_{2}=1 /(1-\lambda)
$$


- de même, les coordonnées linéaires de q relativement à (ca) et relativement à (ac) et celles de $\mathrm{r}$ relativement à $(\mathrm{ab})$ et à $(\mathrm{ba})$,

- avec le théorème du cosinus généralisé, $|\mathrm{pq}|^{2},|\mathrm{qr}|^{2}$ et $|\mathrm{rp}|^{2}$,

$-C\{\mathrm{pqr}\}=|\mathrm{qr}|^{4}+|\mathrm{rp}|^{4}+|\mathrm{pq}|^{4}-2|\mathrm{rp}|^{2}|\mathrm{pq}|^{2}-2|\mathrm{pq}|^{2}|\mathrm{qr}|^{2}-2|\mathrm{qr}|^{2}|\mathrm{rp}|^{2}$.

On trouve (avec le LCF...)

$$
C\{\mathrm{pqr}\}=C\{\mathrm{abc}\} \frac{(\lambda \mu \nu-1)^{2}}{(\lambda-1)^{2}(\mu-1)^{2}(\nu-1)^{2}} .
$$

\section{Corollaire 11}

1. Les points $\mathrm{p}, \mathrm{q}, \mathrm{r}$ sont colinéaires si, et seulement si $\lambda \mu \nu=1$ (le théorème de Ménélaüs).

2. Les côtés du triangle $\{\mathrm{abc}\}$ coupent une droite ne contenant aucun de ses sommets en 0 ou 2 points (l'axiome de Pasch).

Preuve. On contemple le facteur $(\lambda \mu \nu-1)$, et les signes de $\lambda, \mu, v$ si $\lambda \mu \nu=1$.

Les axiomes HIII1, HIII2 et HIII3 se prouvent en définissant la longueur d'un segment comme la distance de ses extrémités et en remplaçant la congruence par l'égalité des longueurs.

L'axiome HIII4 est plus difficile; il faut définir le demi-plan [4].

Définition 9 Soit $\mathrm{D}$ une droite et a un point hors de $\mathrm{D}$. Le demi-plan fermé de frontière $\mathrm{D}$ ne contenant pas a est l'ensemble des points $\mathrm{x}$ tels que le segment fermé $\mathrm{S}_{[\mathrm{ax}]}$ coupe $\mathrm{D}$.

Si l'on remplace dans cette définition a par un point du même côté de $\mathrm{D}$, les demi-plans définis restent les mêmes, à cause de l'axiome de Pasch.

Pour prouver l'axiome HIII4, je choisis ad libitum une demi-droite $\mathrm{D}_{[\mathrm{ab} \infty}$, un angle $\alpha \in$ $[0, \pi]$ et l'un des demi-plans de frontière $\mathrm{D}_{\mathrm{ab}}$. Je construis ensuite une demi-droite de sommet a contenue dans le demi-plan choisi et formant avec $\mathrm{D}_{[\mathrm{ab} \infty}$ un angle de mesure $\alpha$. Voyons le détail (Fig. 2):

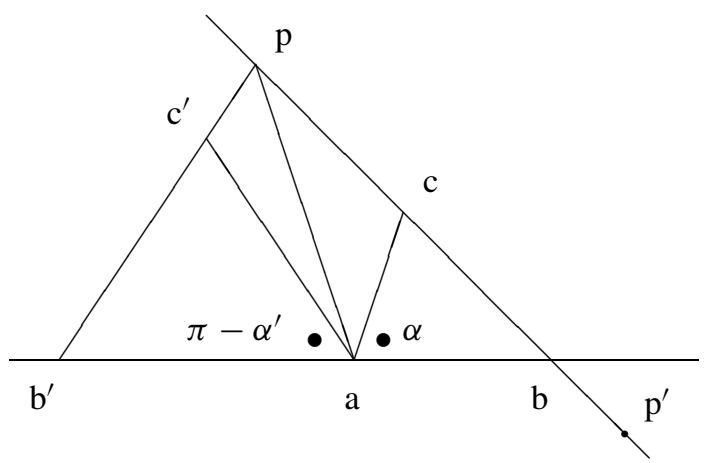

Fig. 2 
- C'est seulement à ce stade qu'il faut faire appel à l'axiome 6: il existe un point $\mathrm{p}^{\prime}$ hors de la droite $\mathrm{D}_{\mathrm{ab}}$.

- Si p' n'est pas dans le demi-plan choisi, je le remplace par un point $\mathrm{p}$ de la demidroite $\mathrm{D}_{\left[\mathrm{p}^{\prime} \mathrm{b} \infty\right.}$ situé au-delà du point $\mathrm{b}$. Sinon, j'appelle $\mathrm{p}$ le point $\mathrm{p}^{\prime}$.

- Si $\alpha \leq \mu(\mathrm{pab})$, je choisis sur le segment $\mathrm{S}_{[\mathrm{bp}]}$ l'unique point $\mathrm{c}$ tel que $\mu(\mathrm{bac})=\alpha$ (voir le lemme 12 infra).

- Si $\alpha \geq \mu$ (pab), je le rebaptise $\alpha^{\prime}$ pour la clarté du dessin. Je remplace ensuite b par un point $\mathrm{b}^{\prime}$ tel que (bab') soit un alignement, et $\alpha$ par $\pi-\alpha^{\prime}$. Puis je reprends la construction précédente.

- La demi-droite cherchée est $\mathrm{D}_{[\mathrm{ac} \infty}$ ou $\mathrm{D}_{\left[\mathrm{ac}^{\prime} \infty\right.}$.

Lemme 12 Avec les notations précédentes, les applications

$$
\begin{aligned}
\mathrm{D}_{[\mathrm{bp} \infty} & \longrightarrow \mathbb{R}_{\geq 0} \longrightarrow[0, \pi-\mu(\mathrm{pba})[ \\
\mathrm{x} & \longmapsto|\mathrm{bx}|
\end{aligned}
$$

et leur composée $f: \mathrm{x} \mapsto \mu(\mathrm{bax})$ sont bijectives.

Preuve. Il suffit de s'occuper de la composée $f$. Soit $\left(\mathrm{bxx}^{\prime}\right)$ un alignement sur la demidroite $\mathrm{D}_{[\mathrm{bp} \infty}$. Comme les points a, $\mathrm{x}, \mathrm{x}^{\prime}$ ne sont pas colinéaires, ni $C\left\{\operatorname{axx}^{\prime}\right\}\left(\operatorname{ni} \sigma\left\{\mathrm{axx}^{\prime}\right\}\right.$ par conséquent) ne sont nuls:

$$
0 \neq \sigma\left\{\operatorname{axx}^{\prime}\right\}=\frac{1}{2}|\operatorname{ax}|\left|\operatorname{ax}^{\prime}\right| \sin \left(\operatorname{xax}^{\prime}\right) .
$$

Donc $\sin \left(\mathrm{xax}^{\prime}\right)$ et $\mu\left(\mathrm{xax}^{\prime}\right)$ sont strictement positifs. Avec le théorème 8.3 , il s'ensuit que $f$ est strictement croissante. Elle est aussi continue car si $\left|\mathrm{xx}^{\prime}\right|<\frac{1}{2}|\mathrm{ax}|$, alors

$$
1-\cos \left(\mathrm{xax}^{\prime}\right)=\frac{\left|\mathrm{xx}^{\prime}\right|^{2}-\left(|\mathrm{ax}|-\left|\mathrm{ax}^{\prime}\right|\right)^{2}}{2|\operatorname{ax}|\left|\mathrm{ax}^{\prime}\right|} \leq \frac{\left|\mathrm{xx}^{\prime}\right|^{2}}{2|\operatorname{ax}|\left(|\mathrm{ax}|-\left|\mathrm{xx}^{\prime}\right|\right)}<\left(\frac{\left|\mathrm{xx}^{\prime}\right|}{|\mathrm{ax}|}\right)^{2}
$$

et $\cos \left(\mathrm{xax}^{\prime}\right)$ tend vers 1 quand $\left|\mathrm{xx}^{\prime}\right|$ tend vers 0 .

Il reste l'axiome des parallèles HIV1 et les axiomes de continuité HV. Suivant une remarque de [4], l'axiome HIV1 est une conséquence des autres axiomes et du théorème 6.2. Les axiomes HV découlent de «mon» axiome 4 et des propriétés de $\mathbb{R}$.

\section{Annexe: Les quinze axiomes plans de Hilbert}

Le cadre de l'axiomatique est un ensemble $\mathrm{P}$ de points, un ensemble $\mathrm{D}$ de droites et une relation $\mathrm{P} \times \mathrm{D} \rightarrow\{$ oui, non\} appelée relation d'incidence. L'incidence est exprimée par les mots «passe par», «contient», «se trouve sur», et caetera.

L'axiomatique originale de Hilbert est celle de la géométrie tridimensionnelle. La dimension 3 est fixée par les axiomes HI7 et HI8. Pour limiter la dimension à deux, il suffit de supprimer les axiomes HI4, HI5, HI6, HI7, HI8 et les références au(x) plan(s) dans l'axiome de Pasch HII4, ainsi que dans HIII4 et HIV1. 
Voici les axiomes de Hilbert:

\section{HI: Axiomes d'incidence}

1. Par deux points donnés passe une droite au moins.

2. Par deux points donnés passe une droite au plus.

3. Une droite contient au moins deux points.

Il existe au moins trois points non colinéaires.

\section{HII: Axiomes d'ordre}

Il existe une relation alignement $\mathrm{P}^{3} \rightarrow$ \{oui, non\}. Si la valeur du triple de points (abc) est oui, on dit que b est situé entre a et $\mathrm{c}$.

1. Si un point $\mathrm{b}$ se trouve entre un point a et un point $\mathrm{c}$, alors $\mathrm{a}, \mathrm{b}, \mathrm{c}$ sont colinéaires et $\mathrm{b}$ se trouve aussi entre $\mathrm{c}$ et a.

2. Etant donné deux points a et $\mathrm{c}$, il existe au moins un point $\mathrm{b}$ de la droite $\mathrm{D}_{\mathrm{ac}}$ tel que $c$ soit situé entre $a$ et $b$.

3. Etant donné trois points colinéaires, l'un d'entre eux au plus est situé entre les deux autres.

4. (Axiome de Pasch.) Soit a, b, c trois points non colinéaires et D une droite ne contenant aucun de ces points. Si $D$ contient un point du segment $S_{[a b]}$, elle contient aussi un point entre a et c ou entre b et c. Le ou n'est pas exclusif.

\section{Définitions}

1. Le segment $\mathrm{S}_{[\mathrm{ab}]}$ est constitué de $\mathrm{a}, \mathrm{b}$ et de l'ensemble des points $\mathrm{x}$ situés entre a et b.

2. La demi-droite $\mathrm{D}_{[\mathrm{ab} \infty}$ est constituée du segment $\mathrm{S}_{[\mathrm{ab}]}$ et de l'ensemble des points $\mathrm{x}$ tels que $\mathrm{b}$ est situé entre a et $\mathrm{x}$.

3. La réunion $\mathrm{H} \cup \mathrm{K}$ de deux demi-droites distinctes $\mathrm{H}, \mathrm{K}$ de même sommet a est un angle. On désigne cet angle par $\angle \mathrm{HK}$ ou par $\angle \mathrm{hak}$, h étant un point de $\mathrm{H}$ et $\mathrm{k}$ un point de $\mathrm{K}$.

4. Un ensemble fini de $n$ points est une figure. Une figure détermine $\frac{1}{2} n(n-1)$ segments et $\frac{1}{2} n(n-1)(n-2)$ angles. Ce sont les segments et les angles de la figure.

\section{HIII: Axiomes de congruence}

La congruence est une relation d'équivalence (notée $\equiv$ ) entre les segments et entre les angles, étendue ensuite aux figures. Deux figures $\mathrm{F}$ et $\mathrm{F}^{\prime}$ sont congruentes (on écrit $\mathrm{F} \equiv \mathrm{F}^{\prime}$ ) s'il existe une bijection de l'une vers l'autre et si tous les segments et tous les angles déterminés par des points correspondants sont congruents.

1. Etant donné un segment $\mathrm{S}_{[\mathrm{ab}]}$ et une demi-droite $\mathrm{D}_{\left[\mathrm{a}^{\prime} \mathrm{c}^{\prime} \infty\right.}$, il existe au moins un point $\mathrm{b}^{\prime}$ sur $\mathrm{D}_{\left[\mathrm{a}^{\prime} \mathrm{c}^{\prime} \infty\right.}$ tel que $\mathrm{S}_{[\mathrm{ab}]} \equiv \mathrm{S}_{\left[\mathrm{a}^{\prime} \mathrm{b}^{\prime}\right]}$.

2. Les congruences $S_{\left[a^{\prime} b^{\prime} \mid\right.} \equiv S_{[a b]}$ et $S_{\left[a^{\prime \prime} b^{\prime \prime}\right]} \equiv S_{[a b]}$ impliquent $S_{\left[a^{\prime} b^{\prime}\right]} \equiv S_{\left[a^{\prime \prime} b^{\prime \prime}\right]}$.

3. Soit $b$ un point entre les points a et $c$ et $b^{\prime}$ un point entre les points $a^{\prime}$ et $c^{\prime}$. Si $S_{[a b \mid} \equiv S_{\left[a^{\prime} b^{\prime}\right]}$ et $S_{[b c]} \equiv S_{\left[b^{\prime} c^{\prime}\right]}$, alors $S_{[a c]} \equiv S_{\left[a^{\prime} c^{\prime}\right]}$. 
4. Etant donné un angle $\mathrm{H} \cup \mathrm{K}$, une demi-droite $\mathrm{H}^{\prime}$ et l'un des demi-plans déterminés par $\mathrm{H}^{\prime}$, il existe exactement une demi-droite $\mathrm{K}^{\prime}$ contenue dans ce demi-plan telle que $\mathrm{H} \cup \mathrm{K} \equiv \mathrm{H}^{\prime} \cup \mathrm{K}^{\prime}$.

5. Si $\mathrm{S}_{[\mathrm{ab}]} \equiv \mathrm{S}_{\left[\mathrm{a}^{\prime} \mathrm{b}^{\prime}\right]}, \mathrm{S}_{[\mathrm{ac}]} \equiv \mathrm{S}_{\left[\mathrm{a}^{\prime} \mathrm{c}^{\prime}\right]}$ et $\angle \mathrm{bac} \equiv \angle \mathrm{b}^{\prime} \mathrm{a}^{\prime} \mathrm{c}^{\prime}$, alors $\angle \mathrm{abc} \equiv \angle \mathrm{a}^{\prime} \mathrm{b}^{\prime} \mathrm{c}^{\prime}$. (Deuxième cas d'égalité)

\section{HIV: L'axiome des parallèles}

1. Etant donné une droite $\mathrm{D}$ et un point a, il existe au plus une droite passant par a et ne coupant pas D.

\section{HV: Axiomes de continuité}

1. Soit deux segments $\mathrm{S}_{[\mathrm{ab}]}$ et $\mathrm{S}_{[\mathrm{cd}]}$. Il existe un entier naturel $n$ tel que $n$ reports du segment $\mathrm{S}_{\text {[cd] }}$ sur la demi-droite $\mathrm{D}_{\text {[ab } \infty}$ à partir du point a conduisent à un point situé au-delà du point b. (Axiome archimédien)

2. Si un ensemble de points d'une droite vérifie tous les axiomes précédents (à l'exception évidente de HIV1), cet ensemble contient tous les points de cette droite: on ne peut pas rajouter de points sans violer l'un des axiomes. (Axiome de complétude linéaire)

\section{Bibliographie}

[1] Berger, M.: Géométrie. Nathan, Paris 1979.

[2] Blumenthal, L.: Theory and Applications of Distance Geometry. Chelsea, New York 1970.

[3] Coxeter, H.S.M.: Geometry. Wyley, New York 1969.

[4] Hilbert, D.: Grundlagen der Geometrie. Teubner, Stuttgart 1962.

[5] Lang, S.: Analysis I. Addision-Wesley, Reading, Massachusetts 1973.

Christoph Soland

Ch. de la Chavanne 14

CH-1092 Belmont-sur-Lausanne

Suisse 